\title{
Pesquisa de Cardiotoxicidade em Prontuário de Paciente Submetido à Quimioterapia R-CHOP: um Estudo de Caso
}

https://doi.org/10.32635/2176-9745.RBC.2019v65n3.314

\author{
Research on Cardiotoxicity in Patient Chart Submitted to R-CHOP Chemotherapy: a Case Study \\ Investigación de Cardiotoxicidad en Prontuario de Paciente Sometido a la Quimioterapia R-CHOP: un Estudio de Caso
}

Patricia Marques Soares Valente'; Eduardo Pinho Braga²; Thamires Ferreira Neves ${ }^{3}$; Walter Claudino Pires de Souza ${ }^{4}$; Thamires Lopes da Silva ${ }^{5}$; Wolney de Andrade Martins ${ }^{6}$; Selma Rodrigues de Castilho ${ }^{7}$

Resumo

Introdução: A cardiotoxicidade é um dos efeitos mais significativos no tratamento oncológico. Entre as implicações mais consideráveis, destaca-se a insuficiência cardíaca com disfunçáo ventricular sistólica ou diastólica. Relato do caso: Paciente do sexo feminino, 39 anos, com linfoma não Hodgkin difuso de grandes células B, submetida à quimioterapia com rituximabe, ciclofosfamida, doxorrubicina, vincristina e prednisona (R-CHOP), que apresentou cansaço aos pequenos esforços e aumento da pressão arterial. O ecocardiograma demonstrou disfunção ventricular esquerda leve com hipocinesia difusa e redução da fração de ejeção do ventrículo esquerdo (FEVE), associadas à possível cardiomiotoxicidade da doxorrubicina. $\mathrm{O}$ caso foi identificado em uma pesquisa de prontuário e analisado segundo o algoritmo de Naranjo e, em seguida, foi determinada a gravidade das reaçóes adversas cardiovasculares. Conclusáo: Este estudo ressalta a importância da busca ativa de eventos cardiovasculares em prontuários de pacientes submetidos à quimioterapia cardiotóxica e do papel da farmacovigilância nesse contexto.

Palavras-chave: Cardiotoxicidade, Doxorrubicina/uso terapêutico; Farmacovigilância; Registros Médicos; Relatos de Casos.

\begin{abstract}
Introduction: Cardiotoxicity is one of the most significant effects of oncological treatment. Among the major relevant consequences, heart failure with systolic or diastolic ventricular dysfunction stands out. Case report: 39-year-old female patient with diffuse large B-cell non-Hodgkin's lymphoma who underwent rituximab, cyclophosphamide, doxorubicin, vincristine and prednisone (R-CHOP chemotherapy) and presented fatigue while exerting small efforts and increased blood pressure. The echocardiogram showed mild left ventricular dysfunction with diffuse hypokinesia and reduction of left ventricular ejection fraction (LVEF) associated with the possible cardiomyotoxicity of doxorubicin. The case was identified in a chart survey, and analyzed according to the Naranjo Algorithm, and then the severity of the adverse cardiovascular reactions was determined. Conclusion: This study highlights the importance of the active search for cardiovascular events in medical charts of patients undergoing cardiotoxic chemotherapy and the role of pharmacovigilance in this context. Key words: Cardiotoxicity; Doxorubicin/therapeutic use; Pharmacovigilance; Medical Records; Case Reports.
\end{abstract}

Resumen

Introducción: La cardiotoxicidad es uno de los efectos más significativos del tratamiento oncológico. Entre los efectos más considerables, destaca la insuficiencia cardíaca con disfunción ventricular sistólica o diastólica.

Relato del caso: Paciente de 39 años con linfoma no Hodgkin difuso de células grandes $\mathrm{B}$ que se sometieron a quimioterapia con rituximab, ciclofosfamida, doxorubicina, vincristina y prednisona (R-CHOP). Pequeńos esfuerzos y aumento de la presión arterial. El ecocardiograma mostró una disfunción ventricular izquierda leve con hipocinesia difusa y reducción de la fracción de eyección del ventrículo izquierdo (FEVI) asociada con la posible cardiomioxicidad de la doxorrubicina. El caso se identificó en una encuesta gráfica y se analizó de acuerdo con el algoritmo de Naranjo, y luego se determinó la gravedad de las reacciones cardiovasculares adversas. Conclusión: Este estudio destaca la importancia de la búsqueda activa de eventos cardiovasculares en los registros médicos de pacientes sometidos a quimioterapia cardiotóxica y el papel de la farmacovigilancia en este contexto. Palabras clave: Cardiotoxicidad; Doxorubicina/uso terapéutico; Farmacovigilancia; Registros Médicos; Informes de Casos.

${ }^{1}$ Programa de Pós-Graduação em Ciências Aplicadas a Produtos para a Saúde. Universidade Federal Fluminense (UFF). Niterói (RJ), Brasil. Orcid iD: https//orcid. org/ 0000-0002-6339-2385

${ }^{2}$ Faculdade de Medicina da UFF. Niterói (RJ), Brasil. Orcid iD: https//orcid.org /0000-0002-5317-5563

${ }^{3}$ Faculdade de Farmácia da UFF. Niterói (RJ), Brasil. Orcid iD: https//orcid.org/0000-0003-1689-5681

${ }^{4}$ Faculdade de Farmácia da UFF. Niterói (RJ), Brasil. Orcid iD: https//orcid.org/0000-0001-7567-7460

${ }^{5}$ Faculdade de Farmácia da UFF. Niterói (RJ), Brasil. Orcid iD: https//orcid.org/0000-0002-8286-6355

${ }^{6}$ Faculdade de Medicina da UFF. Niterói (RJ), Brasil. Orcid iD: https//orcid.org /0000-0002-2284-8251

${ }^{7}$ Faculdade de Farmácia da UFF. Niterói (RJ), Brasil. Orcid iD: https//orcid.org/0000-0003-0272-4777

Endereço para correspondência: Departamento de Farmácia e Administração Farmacêutica da Faculdade de Farmácia da UFF. Rua Mario Vianna, 523 - Santa Rosa. Niterói (RJ), Brasil. CEP: 24241-000. E-mail:selmarc@id.uff.br 


\section{INTRODUÇÃO}

Os linfomas são transformações neoplásicas de células linfoides normais ${ }^{1}$. Se dividem morfologicamente em linfoma de Hodgkin e linfoma não Hodgkin ${ }^{1}$. Os tipos mais comuns de linfoma não Hodgkin em adultos são o linfoma difuso de grandes células $\mathrm{B}$, geralmente agressivo, e linfoma folicular, normalmente indolente ${ }^{1-3}$.

No Brasil, estimam-se 5.370 casos novos de linfoma não Hodgkin em homens e 4.810 em mulheres, para cada ano do biênio 2018-2019 . Para ambos os casos, é a $11^{\text {a }}$ neoplasia mais frequente entre todos os tipos de câncer ${ }^{1}$. Esses valores correspondem a um risco estimado de 5,19 casos novos a cada 100 mil homens e 4,55 casos novos para cada 100 mil mulheres ${ }^{4}$.

A cardiotoxicidade é um dos efeitos mais significativos no tratamento oncológico, e é responsável por grande morbimortalidade, podendo ocorrer de forma aguda ou crônica ${ }^{5}$. A principal manifestação de cardiotoxicidade das terapias oncológicas é a cardiomiopatia relacionada com o uso das antraciclinas (doxorrubicina, epirrubicina, idarrubicina $)^{6,7}$. Estudos mostraram que a disfunção sistólica do ventrículo esquerdo (VE) está relacionada com o uso de doses cumulativas de antraciclinas ${ }^{8}$, administraçóes subsequentes podem resultar em lesōes permanentes tanto celulares quanto intersticiais, que frequentemente estão associadas ao risco de desenvolvimento de insuficiência cardíaca (IC) ${ }^{9}$.

$\mathrm{Na}$ fase aguda, a cardiotoxicidade por antraciclina ocorre até 14 dias após o início do tratamento e é do tipo rara, transitória e independente da dose administrada $^{5}$. Na fase crônica, é dependente da dose e pode ocorrer no período de um ano ou até anos após o fim da quimioterapia ${ }^{5}$. A manifestação mais comum da cardiotoxicidade crônica é a disfunção ventricular sistólica ou diastólica, que pode resultar na cardiomiopatia grave, com risco de morte para o paciente ${ }^{5}$.

Embora já existissem alguns estudos anteriores, foi a partir de 2011, com a publicação da I Diretriz Brasileira de Cardio-Oncologia da Sociedade Brasileira de Cardiologia, que houve maior visibilidade do tema no Brasil ${ }^{5}$. A colaboração e a interação das disciplinas de Cardiologia e Oncologia têm contribuído para reduzir os efeitos adversos cardiovasculares e buscar melhores resultados no tratamento do paciente com câncer ${ }^{5}$.

A farmacovigilância, por meio da busca ativa de reaçôes cardiovasculares em prontuários de pacientes submetidos ao tratamento com quimioterápicos, colabora com a cardio-oncologia sobre o perfil de segurança desses medicamentos. Existe uma lacuna nacional de estudos de farmacovigilância nessa área e o relato apresenta um caso de cardiotoxicidade de uma paciente submetida à terapia com rituximabe, ciclofosfamida, doxorrubicina, vincristina e prednisona (R-CHOP), identificada em um estudo de farmacoepidemiologia.

O objetivo deste artigo é descrever um relato de caso de paciente submetida à quimioterapia com protocolo R-CHOP, de 2013 a 2018, que apresentou cardiotoxicidade após o uso de doxorrubicina.

\section{RELATO DO CASO}

Inicialmente, os dados foram coletados em prontuário. $\mathrm{Na}$ sequência, a paciente foi entrevistada pela farmacêutica para complementação das informaçôes, tendo assinado um Termo de Consentimento Livre e Esclarecido. O trabalho foi aprovado em 14/2/2019 pelo Comitê de Ética em Pesquisa sob o CAAE: 98429018.0.0000.5243.

Paciente do sexo feminino, 39 anos, parda, diarista, natural do Rio de Janeiro, procurou o Serviço de Hematologia de um hospital no Estado do Rio de Janeiro. Sem comorbidade e sem histórico social de tabagismo ou etilismo, diagnosticada com linfoma não Hodgkin difuso de grandes células B no exame histopatológico em 2015, estadiamento III-B, exame prévio de ecocardiograma normal antes do início do tratamento, realizou oito ciclos de quimioterapia R-CHOP. Relatou cansaço aos pequenos esforços em consulta da hematologia, seis meses após o fim da quimioterapia, quando foi realizado um ecocardiograma. Apresentou dimensóes de ventrículo esquerdo normais, porém com perda inicial de geometria elipsoide, e disfunção ventricular esquerda leve, com hipocinesia difusa e sem disfunção segmentar. Função contrátil do ventrículo direito normal. Átrios com dimensôes e volumes normais. Fração de ejeção do ventrículo esquerdo de $45 \%$. O exame foi repetido 15 dias depois com Doppler colorido e apresentou sinais de disfunção diastólica do ventrículo esquerdo em estágio I, com fraçáo de ejeçáo de $70 \%$. Embora o médico tenha relatado a suspeita de que a disfunçáo ventricular e a redução da fração de ejeção estivessem associadas à cardiomiotoxicidade da doxorrubicina, a paciente não iniciou acompanhamento por um cardiologista.

Após o evento relatado, a paciente fez ainda 27 sessóes de quimioterapia, com protocolo ifosfamida, carboplatina e etoposídeo (ICE), e transplante de medula óssea em outra unidade de saúde, e refere boa resposta ao transplante, estando em acompanhamento da hematologia.

A paciente foi entrevistada pela farmacêutica para registro de sinais ou sintomas sugestivos de cardiotoxicidade e relatou cansaço aos pequenos esforços e aumento da pressão arterial após o uso da quimioterapia.

\section{DISCUSSÃO}

A paciente em questão apresentou como fatores de risco para o desenvolvimento de cardiotoxicidade 
por doxorrubicina o sexo feminino, a dose cumulativa maior que $400 \mathrm{mg} / \mathrm{m}^{2}$, e o uso concomitante de outro quimioterápico cardiotóxico, como a ciclofosfamida, o que já foi relatado em outro estudo por Martins et al. ${ }^{10}$. Uma dose total de doxorrubicina igual ou superior a $400 \mathrm{mg} / \mathrm{m}^{2}$ sugere dose cumulativa elevada e necessita

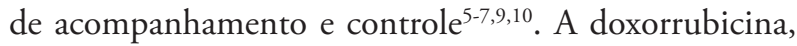
assim como as demais antraciclinas, apresenta uma incidência de cardiotoxicidade (redução da fração de ejeção do ventrículo esquerdo - FEVE e/ou IC) de 5\% a $35 \%$ dos casos e, muitas vezes, irreversível ${ }^{5-7,9,10}$. No caso, a paciente apresentou disfunção ventricular esquerda e fração de ejeção reduzida. Isso resulta da lesão pela doxorrubicina sob o miocárdio ${ }^{10,11}$. As antraciclinas foram descritas inicialmente como dose cumulativa irreversível e por causar lesão mitocondrial, entretanto observou-se a possibilidade de remodelamento reverso e da recuperaçáo da funçáo ventricular ${ }^{10,11}$. No caso apresentado, a paciente teve a recuperação da FEVE.

A partir da entrevista com a farmacêutica, a paciente foi então encaminhada à clínica de IC para iniciar seu acompanhamento. Os eventos cardiovasculares apresentados pela paciente, como o cansaço aos pequenos esforços, o aumento da pressão arterial e a disfunção ventricular esquerda com redução da fraçáo de ejeção do ventrículo esquerdo, foram associados ao uso da doxorrubicina e submetidos à avaliaçáo de causalidade de suspeita de reação adversa pelo algoritmo de Naranjo ${ }^{12}$ (Tabela 1), sendo considerados reações adversas possíveis (escore 4).

No item 5 do algoritmo de Naranjo ${ }^{12}$, foi considerado como causa alternativa: o uso de outro medicamento também cardiotóxico como a ciclofosfamida, que pode causar reduçáo da FEVE e/ou IC de 2\% a $10 \%$ dos $\operatorname{casos}^{13}$. A IC para esse medicamento é incomum $(1 / 1000$ a 1/100) e o aumento da pressão arterial é uma reação muito rara para esse medicamento $(<10 \mathrm{mil})$, isso reduz o valor do algoritmo, porém as reaçôes para doxorrubicina são mais comuns $(5 \% \text { a } 35 \% \text { dos casos })^{13}$.

Em seguida, utilizou-se o Guia para Notificação de Eventos Adversos em Oncologia ${ }^{14}$ para a determinação da gravidade das reaçóes adversas apresentadas. As reaçóes foram consideradas moderadas.

Apesar do uso frequente da doxorrubicina em muitos protocolos de quimioterapia, em razão do seu potencial cardiotóxico, tornam-se necessários o seu acompanhamento e o controle. Medidas como a administração em doses menores em tempos maiores, reduzindo picos plasmáticos, monitoramento de doses acima de $400 \mathrm{mg} / \mathrm{m}^{2}$ e o acompanhamento regular pelo ecocardiograma podem contribuir para evitar a cardiotoxicidade provocada pela doxorrubicina 5 .

Para pacientes com câncer e hipertensão prévia, ou com desenvolvimento de hipertensão após o tratamento do câncer, como no caso relatado, preconiza-se adotar os critérios estabelecidos pelo Eight Joint National Committee $^{15}$ e as recomendaçóes da I Diretriz Brasileira de Cardio-Oncologia 5 . Para esses grupos, recomenda-se o controle e a monitorização da hipertensão arterial durante a quimioterapia e a prevenção dos eventos adversos cardiovasculares para que os pacientes tolerem esse tratamento ${ }^{5,15,16}$.

\section{CONCLUSÃO}

Este resultado mostra a importância da pesquisa de reaçôes cardiovasculares em pacientes que são submetidos à quimioterapia com doxorrubicina, bem como da avaliação regular de doses cumulativas. A farmacovigilância teve

Tabela 1. Determinação da causalidade do cansaço, da disfunção ventricular e da elevação da pressão arterial na exposição à doxorrubicina*

\begin{tabular}{|c|c|c|c|c|}
\hline Questões & Sim & Não & Desconhecido & $\begin{array}{l}\text { Soma dos } \\
\text { escores }\end{array}$ \\
\hline 1. Existem notificações conclusivas sobre essas reações? & +1 & 0 & 0 & +1 \\
\hline 2. A reação apareceu após a administração do fármaco? & +2 & -1 & 0 & +2 \\
\hline 3. A reação melhorou quando o fármaco foi suspenso? & +1 & 0 & 0 & 0 \\
\hline 4. A reação reapareceu quando da sua readministração? & +2 & -1 & 0 & 0 \\
\hline 5. Existem causas alternativas (até mesmo outro fármaco)? & -1 & +2 & 0 & -1 \\
\hline 6. A reação reaparece com a introdução de um placebo? & -1 & +1 & 0 & 0 \\
\hline 7. A concentração plasmática está em nível tóxico? & +1 & 0 & 0 & +1 \\
\hline $\begin{array}{l}\text { 8. A reação aumentou com dose maior ou reduziu com dose } \\
\text { menor? }\end{array}$ & +1 & 0 & 0 & 0 \\
\hline $\begin{array}{l}\text { 9. O paciente experimentou semelhante reação } \\
\text { anteriormente com medicamentos de mesmo fármaco? }\end{array}$ & +1 & 0 & 0 & 0 \\
\hline 10. A reação foi confirmada por qualquer evidência objetiva? & +1 & 0 & 0 & +1 \\
\hline & \multicolumn{3}{|c|}{ Total } & 4 \\
\hline
\end{tabular}

Nota: *Baseado no algoritmo de Naranjo ${ }^{12}$. 
um papel importante na busca ativa, e a aplicação do algoritmo, na determinação da causalidade das reaçóes adversas, colaborando assim para os estudos de cardio-oncologia e no monitoramento de medicamentos. Além disso, a realização do ecocardiograma no monitoramento desses pacientes é igualmente relevante para que se possa assegurar um acompanhamento cardiológico mais precoce.

\section{CONTRIBUIÇÕES}

Patricia Marques Soares Valente, Wolney de Andrade Martins e Selma Rodrigues de Castilho contribuíram substancialmente para a concepção, planejamento, análise e interpretação dos dados, na redação, na revisão crítica e na elaboração da versão final. Eduardo Pinho Braga, Thamires Lopes da Silva, Thamires Ferreira Neves e Walter Claudino Pires de Souza contribuíram substancialmente na análise e interpretação dos dados. Todos os autores aprovaram a versão final a ser publicada.

\section{DECLARAÇÃO DE CONFLITO DE INTERESSES}

Nada a declarar.

\section{FONTES DE FINANCIAMENTO}

Fundação de Amparo à Pesquisa do Estado do Rio de Janeiro (Faperj) e Conselho Nacional de Pesquisa e Desenvolvimento (CNPq).

\section{REFERÊNCIAS}

1. Sánchez-Beato M, Sánchez-Aguilera A, Piris MA. Cell cycle deregulation in B-cell lymphomas. Blood. 2003;101(4):1220-35. doi: https://doi.org/10.1182/ blood-2002-07-2009

2. Macintyre E, Willerford D, Morris SW. Non-Hodgkin's lymphoma: molecular features of B cell lymphoma. Hematology Am Soc Hematol Educ Program. 2000;(1)180-204. doi: https://doi.org/10.1182/ asheducation.V2000.1.180.20000180

3. Araújo LHL, Victoriano APOS, Melo AC, et al. Linfoma não-Hodgkin de alto grau: revisão de literatura. Rev Bras Cancerol. 2008;54(2):175-83.

4. Instituto Nacional de Câncer José Alencar Gomes da Silva. Estimativa 2018: incidência de câncer no Brasil [Internet]. Rio de Janeiro: INCA; 2017. [acesso 2019 abr. 06]. Disponível em: http://www1.inca.gov.br/ estimativa/2018/estimativa-2018.pdf

5. Kalil Filho R, Hajjar LA, Bacal F, et al. I Diretriz Brasileira de Cardio-Oncologia da Sociedade Brasileira de Cardiologia. Arq Bras Cardiol. 2011;96(2 Supl $1): 1-52$.
6. Adão R, Keulenaer G, Leite-Moreira A, et al. Cardiotoxicidade associada à terapêutica oncológica: mecanismos fisiopatológicos e estratégias de prevenção. Rev Port Cardiol. 2013;32(5):395-409. doi: https://doi. org/10.1016/j.repc.2012.11.002

7. Ewer MS, Ewer SM. Cardiotoxicity of anticancer treatments: what the cardiologist needs to know. Nat Rev Cardiol.2010;7(10):564-75. doi: https://doi. org/10.1038/nrcardio.2010.121

8. Lefrak EA, Pitha J, Rosenheim S, et al. A clinicopathologic analysis of adriamycin cardiotoxicity. Cancer. 1973;32(2):302-14. doi: https://doi. org/10.1002/1097-0142(197308)32:2<302::AIDCNCR2820320205>3.0.CO;2-2

9. Albini A, Pennesi G, Donatelli F, et al. Cardiotoxicity of anticancer drugs: the need for cardio-oncology and cardio-oncological prevention. J Natl Cancer Inst.2010;102(1):14-25. doi: https://doi.org/10.1093/ jnci/djp440

10. Martins WA, Souza VB, Rocha LFC, et al. Cardiomiotoxicidade por doxorrubicina em pacientes com câncer de mama: a propósito de dois casos. Rev Bras Cardiol. 2011; 24(3):196-98.

11. Cardinale D, Colombo A, Lamantia G, et al. Anthracycline-induced cardiomyopathy: clinical relevance and response to pharmacologic therapy. J Am Coll Cardiol. 2010;55(3):213-20. doi: https://doi. org/10.1016/j.jacc.2009.03.095

12. Naranjo CA, Busto U, Sellers EM, et al. A method for estimating the probability of adverse drug reactions. Clin Pharmacol Ther. 1981;30(2):239-45. doi: https://doi. org/10.1038/clpt.1981.154

13. Genuxal Baxter [Internet]. São Paulo: Baxter Hospitalar Ltda.; 2019. Bula de medicamento. [acesso 2019 nov. 11]. Disponível em: http://www.anvisa.gov.br/datavisa/ fila_bula/frmVisualizarBula.asp?pNuTransacao $=496835$ 2019\&pIdAnexo=11207943

14. Sociedade Brasileira de Farmacêuticos em Oncologia. Guia para notificação de reaçôes adversas em oncologia. 2. ed. Belo Horizonte: ANVISA; 2011.

15. James PA, Oparil S, Carter BL, et al. 2014 Evidencebased guideline for the management of high blood pressure in adults: report from the panel members appointed to the Eighth Joint National Committee (JNC 8). JAMA. 2014;311(5):507-20. doi: https://doi. org/10.1001/jama.2013.284427

16. Souza VB, Silva EN, Ribeiro ML, et al. Hipertensão arterial no paciente com câncer. Arq Bras Cardiol. 2015;104(3):246-52. doi: http://dx.doi.org/10.5935/ abc. 20150011 